\title{
Contemporary use of cardiac imaging for COVID-19 patients: a three center experience defining a potential role for cardiac MRI
}

\author{
Ankur Panchal $^{1} \cdot$ Andreas Kyvernitakis $^{2} \cdot$ J. Ronald Mikolich ${ }^{3} \cdot$ Robert W. W. Biederman $^{2}$
}

Received: 23 September 2020 / Accepted: 17 December 2020 / Published online: 9 February 2021

(c) The Author(s), under exclusive licence to Springer Nature B.V. part of Springer Nature 2021

\begin{abstract}
The pandemic of coronavirus disease 2019 (COVID-19) secondary to the novel severe acute respiratory syndrome coronavirus 2 (SARS-CoV-2) has bestowed an unprecedented challenge upon us, resulting in an international public health emergency. COVID-19 has already resulted in >1,600,000 deaths worldwide and the fear of a global economic collapse. SARS-CoV-2 is notorious for causing acute respiratory distress syndrome, however emerging literature suggests various dreaded cardiac manifestations associated with high mortality. The mechanism of myocardial damage in COVID-19 is unclear but thought to be multifactorial and mainly driven by the host's immune response (cytokine storm), hypoxemia and direct myocardial injury by the virus. Cardiac manifestations from COVID-19 include but are not limited to, acute myocardial injury, cardiac arrhythmias, congestive heart failure and acute coronary syndrome. Cardiac imaging is paramount to appropriately diagnose and manage the cardiac manifestations of COVID-19. Herein, we present cardiac imaging findings of COVID-19 patients with biomarker and imaging confirmed myocarditis to provide insight regarding the variable manifestations of COVID-19 myocarditis via Cardiac MRI (CMR) coupled with CMR-edema education along with recommendations on how to incorporate advanced CMR into the clinicians' COVID-19 armamentarium.
\end{abstract}

Keywords SARS-CoV-2 · COVID-19 · Coronavirus · Cardiac MRI

\section{Background}

Coronaviruses are single stranded positive sense RNA viruses with ability for rapid mutation. There are at least six human susceptible coronaviruses that have been identified, among which Severe Acute Respiratory Syndrome coronavirus (SARS-CoV) and Middle East Respiratory Syndrome coronavirus (MERS-CoV) are known to cause severe acute respiratory distress syndrome. SARS-CoV-2 is a novel coronavirus with similar pathogenicity as SARS-CoV and uses the angiotensin converting enzyme 2 (ACE2) receptors to enter human cells [1]. The outbreak of this novel coronavirus first occurred in December 2019 in Wuhan, China

Robert W. W. Biederman

Robert.Biederman@ahn.org

1 Internal Medicine, University of Pittsburgh Medical Center, Pittsburgh, PA, USA

2 Cardiovascular Disease, Allegheny Health Network, Pittsburgh, PA, USA

3 Sharon Regional Medical Center, Sharon, PA, USA and has spread across the world rapidly. The World Health Organization declared it to be a pandemic in March 2020 [2]. The number of cases has increased over the past eight months and as of now, it has reached $>73$ million infected and $>1,600,000$ deaths across the globe. Although the most common presentation is acute respiratory failure, cardiovascular complications are increasingly reported and are a dreaded manifestation due to increased mortality. While most of the research has so far focused on investigating the causality, pathophysiology, treatment and outcomes of myocarditis in COVID-19 patients, there is paucity of literature on identifying patterns on cardiac imaging in this population. We present cases of COVID-19 myocarditis that have been published with cardiac imaging findings dovetailed with unique aspects of CMR-derived myocardial-edema imaging. 


\section{Mechanism of COVID-19-related cardiovascular disease}

Coronaviruses, such as MERS-CoV are known to cause myocarditis [3]. Due to similarities in pathogenicity of MERS-CoV and SARS-CoV-2, cardiac involvement with SARS-CoV-2 was suspected and first described in Wuhan, China when 5 out of 41 COVID-19 patients were found to have acute myocardial injury [4]. Myocarditis is considered to be an important cause of acute myocardial injury in these patients and has been identified on cardiac autopsies from the National Health Commission PRC, demonstrating myocyte necrosis along with mononuclear leukocyte infiltration in COVID-19 deceased patients [5-7]. Moreover, a study in China on recovered COVID-19 patients who had reported cardiac symptoms revealed that $58 \%$ of them had evidence of myocarditis on cardiac MRI (CMR), implying that the incidence of myocardial involvement with SARS$\mathrm{CoV}-2$ is much more common than previously thought in this population [8]. The mechanism for acute myocardial injury and myocarditis is yet to be fully elucidated but is suspected to be secondary to a severe inflammatory response and cytokine storm, resulting in direct myocardial damage (Fig. 1a) [9, 10]. Similarly, this fulminant cytokine release may cause acute coronary syndrome,
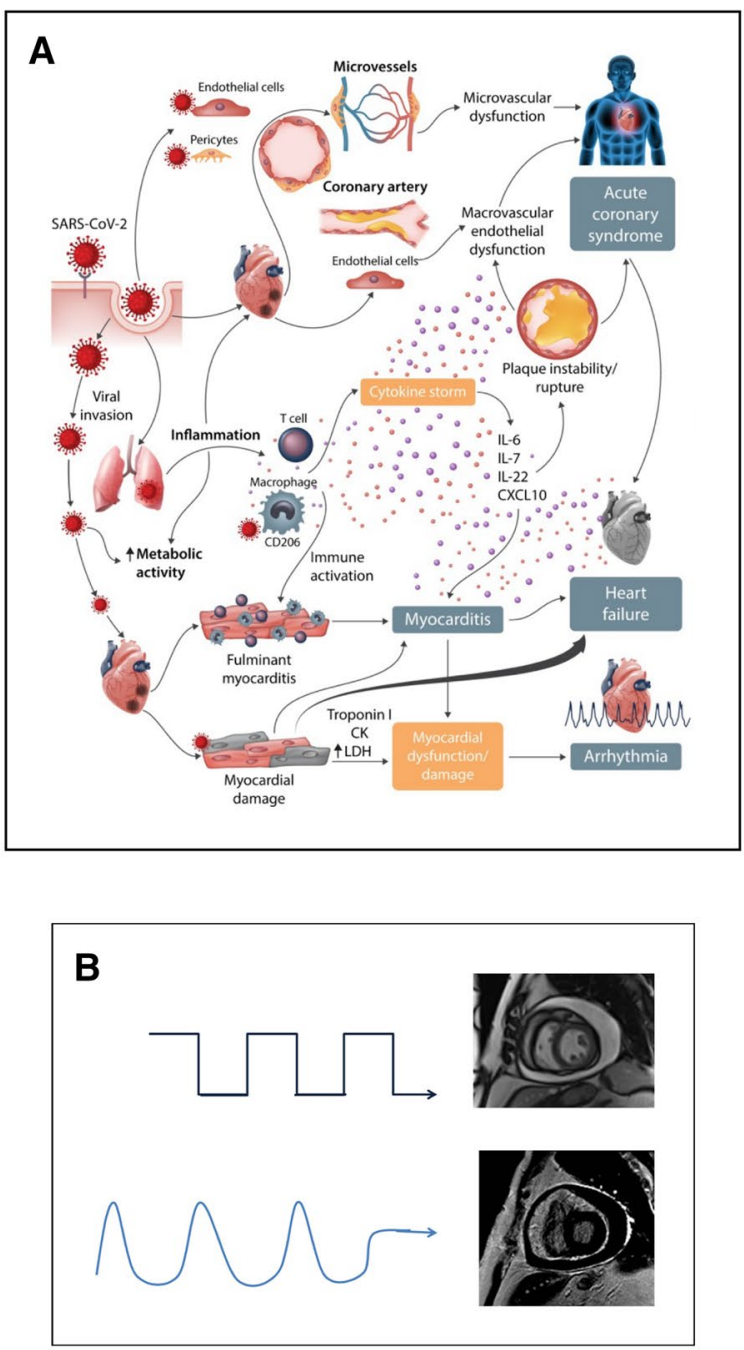
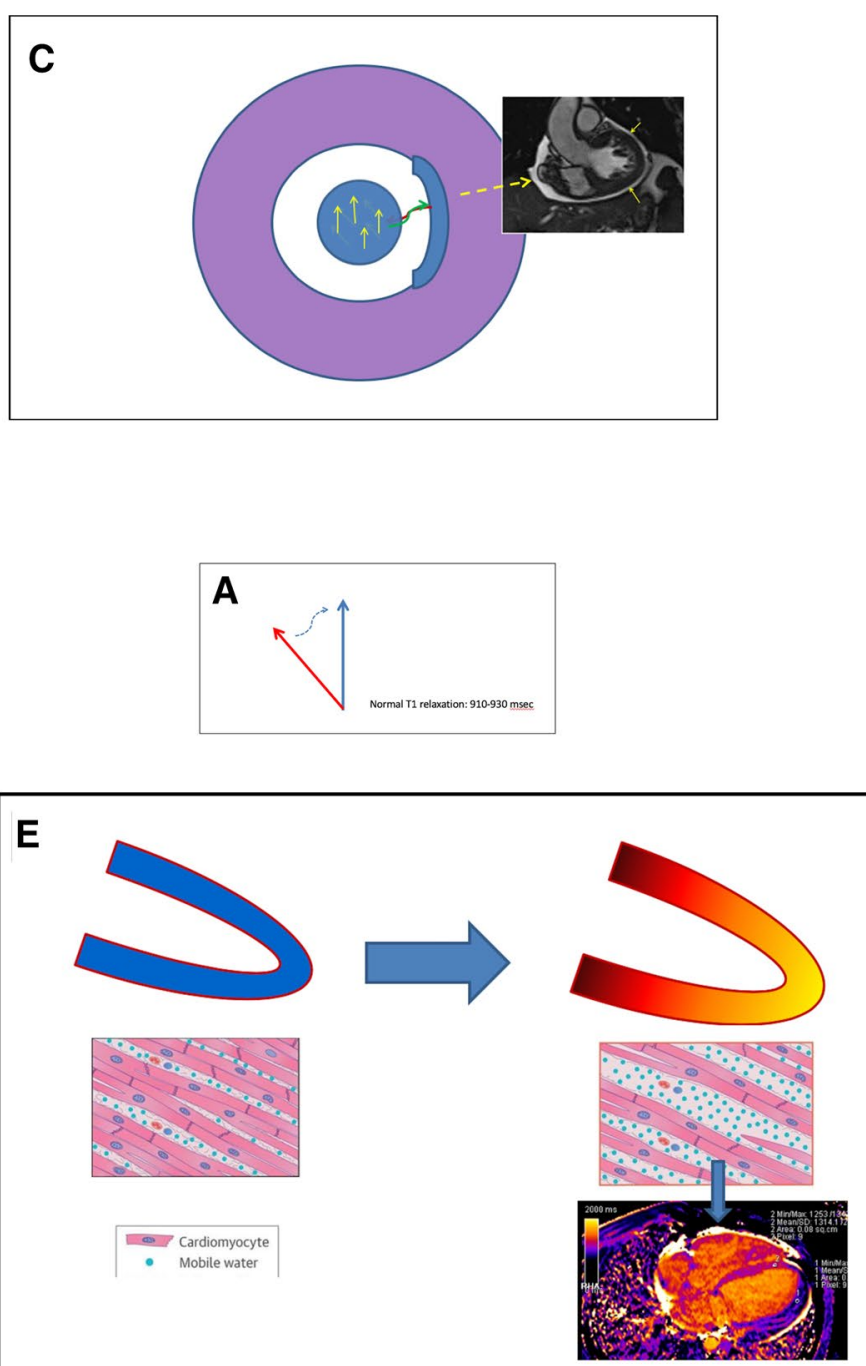

Fig. 1 a Suspected mechanism of COVID-19-related acute myocardial injury. This figure is adopted from European Society of Cardiology, Cardiovascular research with copyright acceptance [10]. b Variance of the physical characteristics of a pulse sequence radio wave alter the appearance of a CMR image. $\mathbf{c}$ Return of a hydrogen pro- ton back to the due North orientation causes a reflected radio wave, which after Fourier transform, generates the CMR image. d The time to restore a hydrogen proton from a flipped angle position back to due North, defines T1 relaxation time. e Increase in myocardial wall water (edema) causing an increase in $\mathrm{T} 1$ relaxation time 
due to increased oxidative stress, coronary plaque destabilization and rupture [11]. Alternative theories include acute respiratory failure causing oxygen supply-demand mismatch to the myocardium and direct viral entry into cardiac myocytes through the ACE2 receptors [12].

\section{Diagnosing COVID-19 myocarditis: use of cardiac imaging}

Myocarditis is defined as inflammation of the cardiac muscle by infectious or noninfectious etiologies [13]. Clinical modalities that are traditionally used to evaluate cardiac injury include cardiac biomarkers (cardiac troponin, creatine kinase MB, C-reactive protein (CRP), N-terminal pro B-type natriuretic peptide (pro-BNP)), non-invasive imaging (electrocardiogram, echocardiogram, nuclear perfusion studies, cardiac CT and CMR), and invasive techniques (coronary angiography, endomyocardial biopsy). Due to the extremely high infectivity rate of this novel coronavirus, careful clinical judgement has been advised by multiple cardiovascular societies on the appropriate use criteria for performing cardiac imaging which is essential in the diagnosis of COVID19 myocarditis [14-18].

Transthoracic echocardiogram (TTE) is the initial noninvasive imaging modality of choice since it can provide valuable data for diagnosing myocardial infarction, aortic root dissection, pericardial disease or valvular heart disease. Common TTE findings in myocarditis include new left ventricular (LV) wall motion abnormalities, diastolic dysfunction, myocardial edema, intraventricular thrombus, and pericardial effusion [19]. Interestingly, recently published study on echocardiographic findings in COVID-19 patients demonstrated that only a third of the hospitalized patients have normal biventricular function. Although LV systolic function was preserved in majority of patients, LV diastolic and RV function were frequently impaired [20]. On the other hand, a negative echocardiogram is inadequate to definitely rule out myocarditis and in such cases, CMR can be highly valuable.

Since the original first dozen case reports there have been many more, we determined to discover pattern of CMR findings in patients with COVID-19 myocarditis [21-24, 6, 25-36]. It is noteworthy that COVID-19 has shown sexual dimorphism with men having higher susceptibility for infection and complications [37-39]. Of the published myocarditis cases that we reviewed [21-24, 6, 25-31], 8 (67\%) were male, though data regarding mortality was limited. TTE was performed in 11 out of 12 patients and interestingly 5 (45\%) were reported to have normal LV systolic function. The most common findings in those with abnormal systolic function included global hypokinesis and moderate to severe LV systolic dysfunction. Acute diastolic dysfunction was reported in one patient and pericardial effusion was present in 3 cases, including one patient with cardiac tamponade requiring emergent pericardiocentesis. Interestingly, nuclear imaging was not performed in any of our discussed case reports.

CMR was performed in 7 out of 12 patients. Short tau inversion recovery and T2-mapping sequences in most of these patients showed significant interstitial myocardial edema. Phase sensitive inversion recovery sequences showed late gadolinium enhancement (LGE) in all but two cases $[6,31,28]$. Interestingly, among the five patients who had normal cardiac function on TTE, three patients $(60 \%)$ had confirmed acute myocarditis by CMR [22, 27, 31]. Thus, in COVID-19 patients who lack typical diagnostic features in their echocardiogram, the diagnosis of myocarditis can be made by CMR, aligning with prior data suggestive of CMR having more diagnostic accuracy in detecting myocarditis [40].

In one patient, echocardiography with dynamic-3D volume reconstruction was utilized and showed normal apical contraction along with hypokinesia of the mid and basal segments of LV suggesting reverse TakoTsubo pattern. CMR showed diffuse myocardial edema and lack of LGE to suggest myocardial scarring. Subsequent endomyocardial biopsy also exhibited diffuse T-cell lymphocytic infiltrates and interstitial edema with limited foci of necrosis. No replacement fibrosis was detected, suggestive of an acute inflammatory process. SARS-CoV-2 genome was not detected in myocardium during molecular analysis, suggesting an acute virus negative lymphocytic myocarditis associated with SARS-CoV-2 respiratory infection [28].

The diagnosis of myocarditis is often difficult, because myocardial inflammation maybe global, regional or patchy. The presumptive "gold standard" for the diagnosis of myocarditis is myocardial biopsy with pathologic evidence of inflammation. However, even biopsy is not reliable if there is no global myocardial cellular involvement. Unlike echocardiography, nuclear imaging and angiography, CMR is capable of "tissue characterization", distinguishing scarring, hemorrhage, infarction, infiltrate and tumor from interstitial myocardial edema [41].

\section{Attributes of CMR}

CMR does not require radiation exposure, but rather uses magnetic properties and radio waves to generate motion images with excellent wide-field spatial resolution. When placed into a magnetic field, the hydrogen protons of myocardial water molecules effectively can be thought to align into a "due North" orientation (Video 1). A "coil" transmitter sends a radio wave of specific physical characteristics (called a "pulse sequence") into the myocardium deflecting 
the hydrogen protons into a "flipped angle" position. When the pulse sequence is terminated, the hydrogen protons immediately go back to "due North" (Video 2), giving off a reflected radio wave which is mathematically converted via a Fourier transform to clinically useful image (Fig. 1b, c). The coil alternates rapidly between transmitter and receiver, to accomplish image acquisition. When coupled with electrocardiographic gating, high quality motion images are generated. By varying the physical properties of the transmitted radio wave pulse sequence, the appearance of cardiac tissues varies, allowing MRI to achieve "tissue characterization" [41, 42].

The workhorse imaging pulse sequence for CMR is steady state free precession (SSFP). SSFP imaging is very useful for assessing structural aspects of myocardial tissue and valve motion. Similar to echocardiography, multiple imaging "slices" are obtained to allow visualization of as many of the cardiac structures as possible. However, CMR has a full field of view and is not limited to a $90^{\circ}$ arc of imaging within the confines of a thoracic intercostal space. This advantage of CMR is critically important in being able to fully assess the right ventricle (RV).

Detection of myocardial edema (an indirect marker of inflammation) by CMR uses assessment of hydrogen proton movement as it is forced into and out of the "flip angle" position. The time it takes the hydrogen proton to return from the "flip angle" position to "due North" position in the $\mathrm{X}-\mathrm{Y}$ axis plane is called the "T1 relaxation time" (Fig. 1d). The time it takes the hydrogen proton to return from the "flip angle" position to "due North" in the Y-Z axis plane is called the "T2 relaxation time". Because $\mathrm{T} 1$ and $\mathrm{T} 2$ relaxation times vary with water content, quantification of these relaxation parameters on a pixel-by-pixel basis, with color encoding, yield valuable clinical information about myocardial edema and possible inflammation (Fig. 1e). Increased myocardial water content (i.e. edema) prolongs both $\mathrm{T} 1$ and $\mathrm{T} 2$ relaxation times. Use of this imaging technique not only allows detection of myocardial edema in myocarditis, but also provides information as to whether the inflammation is global, regional or patchy. If necessary, this information provides valuable guidance for determining an appropriate site for endomyocardial biopsy.

\section{Descriptive cases of COVID-19 myocarditis}

In our recent experience, we encountered four emblematic cases revealing the myriad of COVID-19 myocarditis presentations. The first was a 65 -year old Caucasian male with multiple cardiovascular comorbidities including paroxysmal atrial fibrillation on systemic anticoagulation but preserved LV systolic function, who presented with flulike symptoms and rapidly progressed to acute hypoxemic respiratory failure due to PCR confirmed COVID-19 disease, requiring ventilator support. He was initially treated with hydroxychloroquine, azithromycin and plasmapheresis with clinical improvement and was successfully extubated. Daily troponin $\mathrm{T}$ levels were checked and were normal until day 10 of admission, when they were found to be elevated and up-trending (peak $0.61 \mathrm{ng} / \mathrm{mL}$ ), but interestingly, the patient was hemodynamically stable and remained asymptomatic. Electrocardiogram revealed non-specific $\mathrm{T}$ wave abnormalities in the anterolateral leads and TTE revealed new LV regional wall motion abnormalities consistent with a reverse TakoTsubo pattern with LV ejection fraction (EF) of $40-45 \%$ (Fig. 2). The patient suffered from a pulseless electrical arrest $24 \mathrm{~h}$ later and despite successful return of spontaneous circulation following cardiopulmonary resuscitation, his course was complicated with multiorgan failure and eventually expired. Following his resuscitation, pulmonary embolism was ruled out and his decompensation, given the presentation, was attributed to COVID-19 myocarditis. These authors (we) recently published this interesting case [36].

Another interesting case was a 67-year old Caucasian female with history of hypertension, who presented with symptoms of congestive heart failure (CHF). She was found to have LV systolic dysfunction with mild global hypokinesis on echocardiogram along with elevated troponin I levels $(0.31 \mathrm{ng} / \mathrm{mL})$. CMR revealed LV systolic dysfunction (EF $30 \%$ ) and moderate global RV hypokinesis that was not well visualized by TTE. T1 and T2 relaxation mapping were suggestive of myocardial wall edema and LGE was noted on phase sensitive inversion recovery especially involving the RV which was not appreciated on the TTE. Although she was never considered high-risk for SARS-CoV-2 infection per the CDC guidelines, her PCR was positive for SARSCoV-2, unveiling final diagnosis of acute COVID-19 myocarditis (Fig. 3). With regard to therapy, hydroxychloroquine was not a viable option due to prolongation of the QTc interval. Alternatively, colchicine was initiated due to the substantial biventricular edema and on day nine she was eventually discharged. Colchicine was favored in our patient for an important reason. Hydroxychloroquine is believed to exert its anti-inflammatory effect by interfering with antigen processing, which is required to stimulate $\mathrm{CD} 4+\mathrm{T}$ cells for an auto-immune response, typical of a cytokine storm [43]. Colchicine does not interfere with stimulation of CD4+ T cells but rather affects white blood cells, causing microtubule depolymerization, which severely limits WBC degranulation. In addition, colchicine directly disrupts the NOD-like receptor protein-3 (NLRP3) inflammasome protein complex, which is a critical component of an anti-inflammatory response [44-46]. Moreover, increased resolution of myocarditis, associated with pericarditis, among patients being treated with colchicine was noted by Morgenstern et al. [47]. 
Fig. 2 Apical 4-chamber views and parasternal long axis views demonstrating basal to mid LV systolic dysfunction with preserved distal and apical segments, in a reverse TakoTsubo pattern (images 1-4)
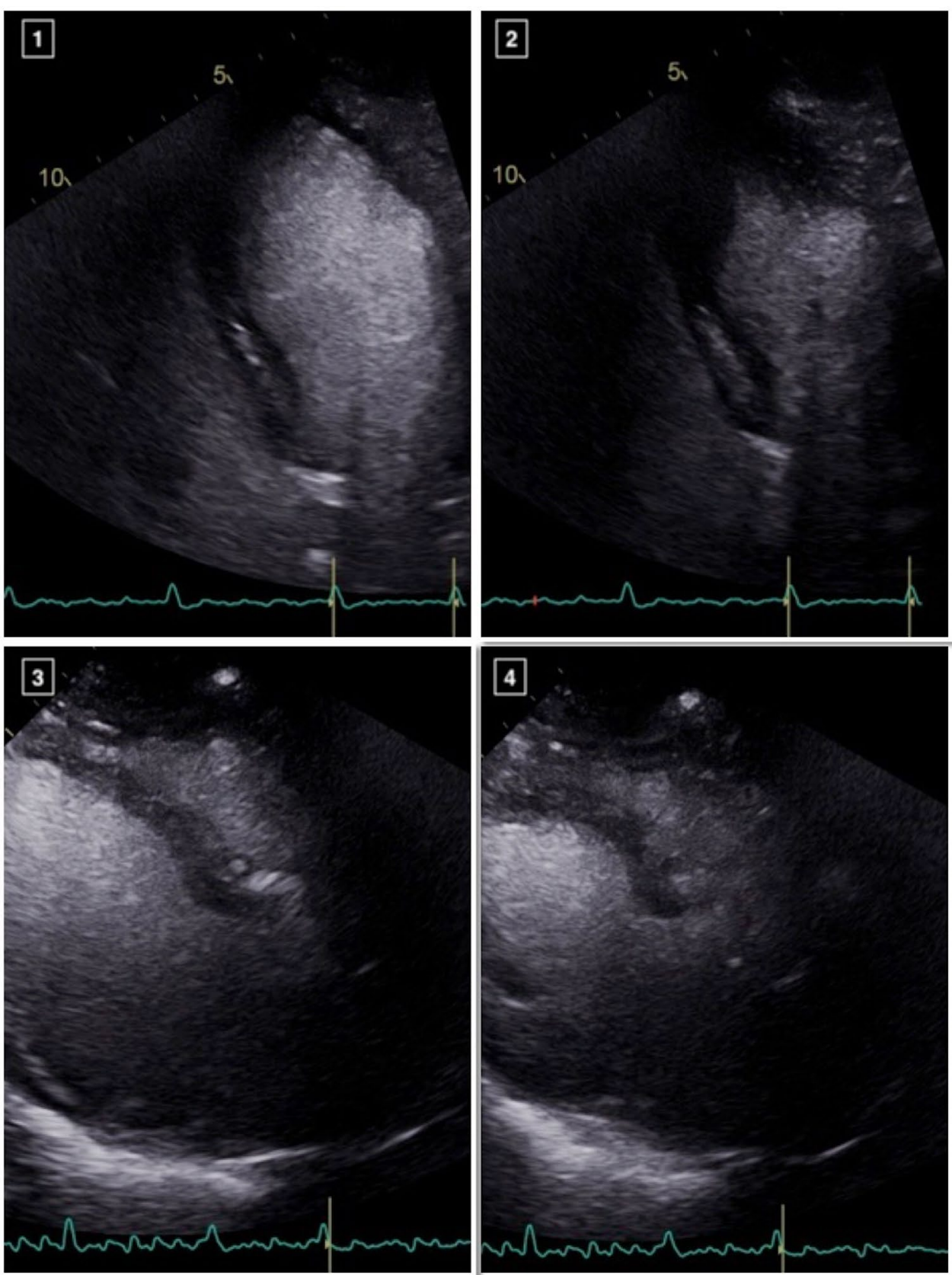

We encountered a 78-year old Caucasian man patient with multiple cardiac comorbidities but preserved LV systolic function at baseline, who was admitted with flu-like symptoms and acute hypoxemic, ventilator-dependent respiratory failure. He was found to be positive for COVID-19 disease. An electrocardiogram revealed ST segment elevation in the anterior leads along with elevation of troponin $\mathrm{T}$ levels to $4.1 \mathrm{ng} / \mathrm{mL}$, and his echocardiogram demonstrated new severe global LV dysfunction with EF of 35\% (Fig. 4). Surprisingly, the epicardial coronary arteries were without significant disease on invasive coronary angiogram. This patient eventually expired due to multi-organ failure.
Lastly, a 72-year old Caucasian man with history of hypertension, hyperlipidemia and type 2 diabetes, who was admitted with CHF, was noted to have asymptomatic rising troponin I (peak $0.14 \mathrm{ng} / \mathrm{mL}$ ), along with anterior T wave inversions on electrocardiogram. Coronary arteriography demonstrated significant stenosis of the proximal LAD and second obtuse marginal branch of the left circumflex artery (LCX), with hypokinetic anterior LV wall, all raising concern about the viability of myocardium in the distribution of LAD. The edema of the LV wall induced by inflammation from COVID-19 myocarditis would increase LV wall tension and thus increase myocardial oxygen demand, potentially 

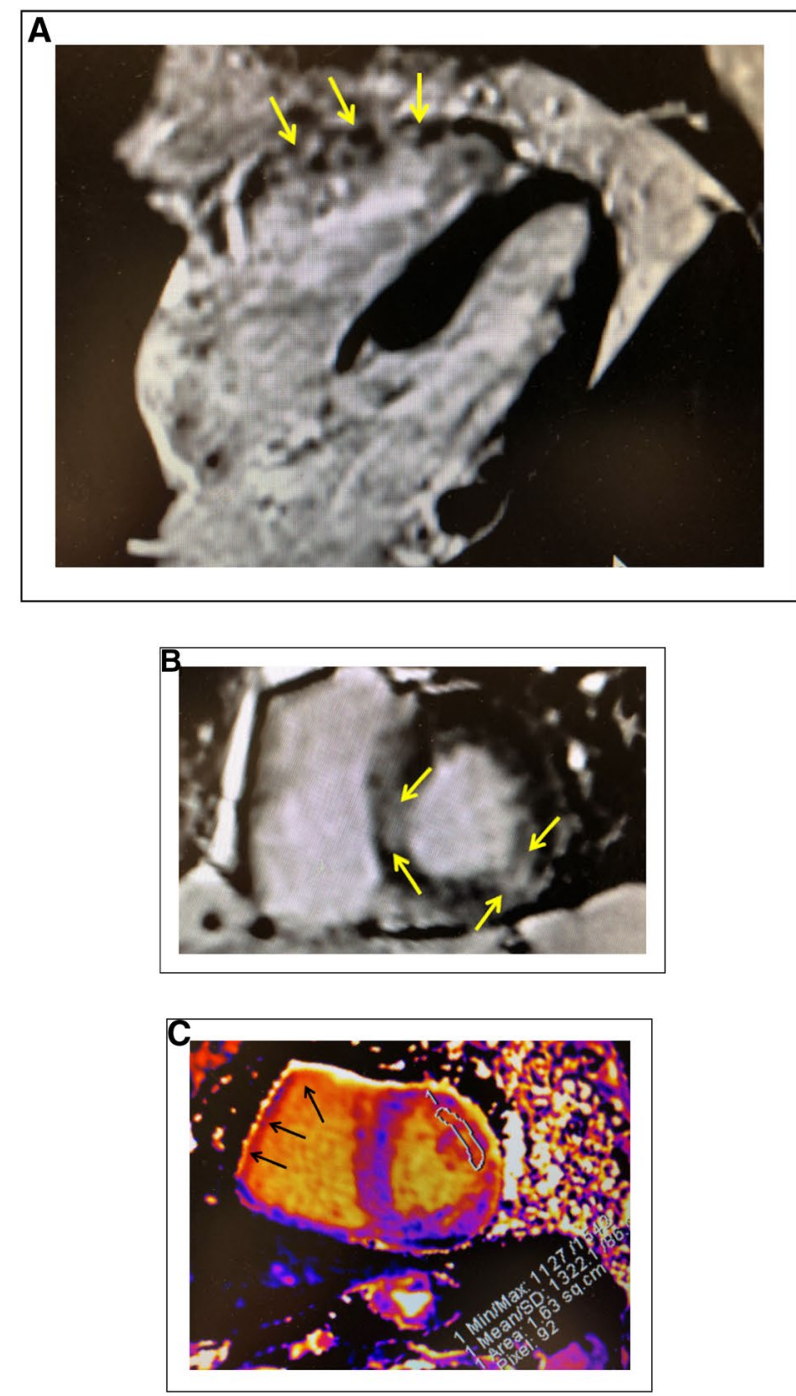

Fig. 3 a Abnormal uptake of gadolinium involving the free wall of the RV (yellow arrows). Compare with the normal appearing LV myocardium on LGE imaging. b Abnormal basal septal and basal inferolateral wall LGE of the LV (yellow arrows) in short axis view. c Edema of the RV free wall (black arrows) and mid anterolateral wall of the LV (Sampled area \#1) with prolongation of the T1 relaxation

exacerbating the ischemia of the underlying coronary atherosclerosis. CMR demonstrated a completely viable myocardium but diffuse myocardial edema on T1 and T2 mapping technique, with global LV hypokinesis (EF 38\%), supportive of myocarditis (Fig. 5). This patient was found to have positive PCR for COVID-19. Here, the CMR was instrumental in redirecting care to COVID-19 myocarditis, not to unnecessary coronary artery bypass grafting $(\mathrm{CABG})$ with the further risk of infecting several health-care workers.

Overall, in our experience, CMR showed more diffuse involvement of myocardium in COVID-19 induced myocarditis compared to non-COVID myocarditis. All of our T1 and $\mathrm{T} 2$ relaxation time values were quantified from Siemens
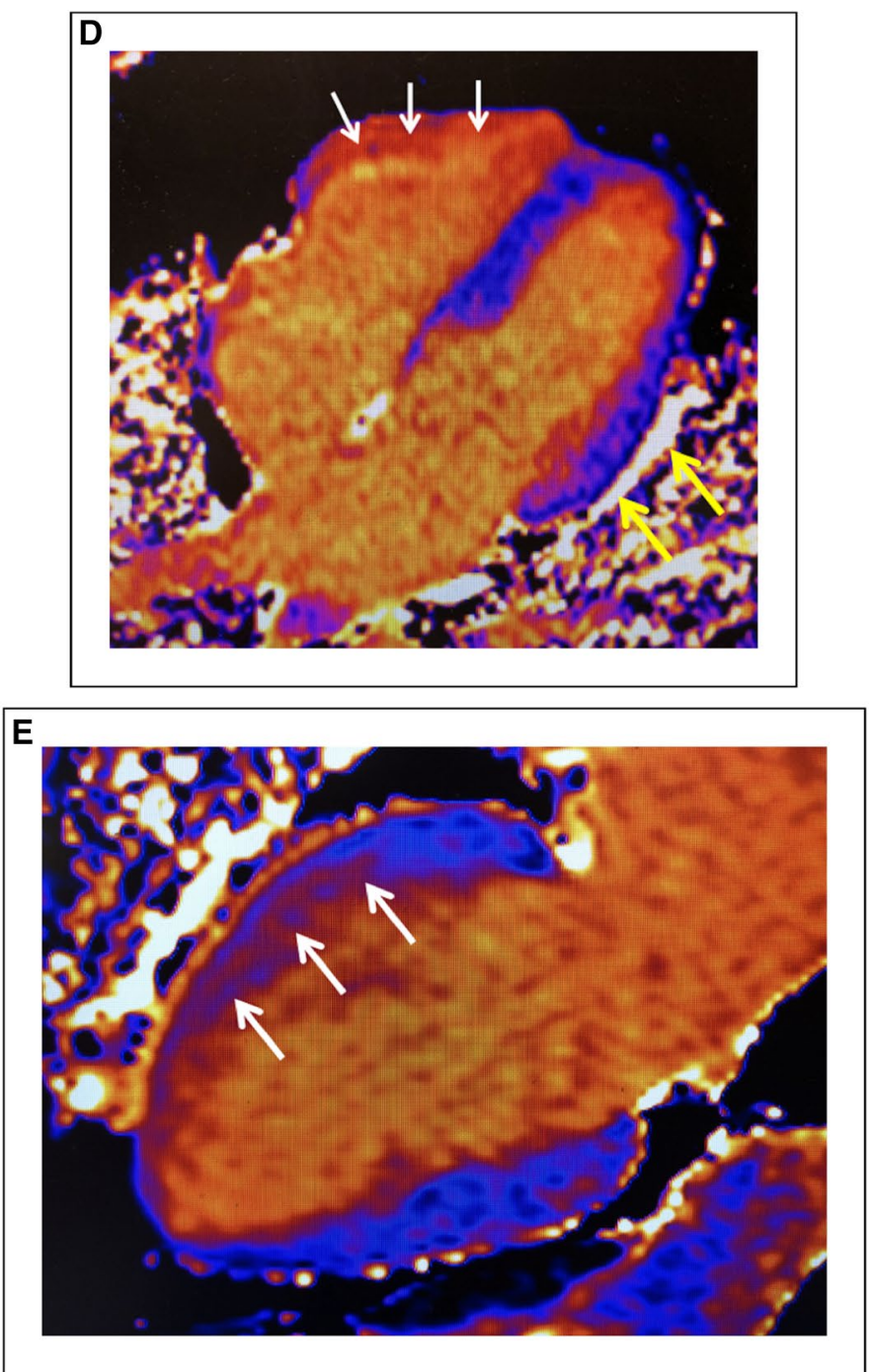

time to 1322 msec. d Diffusely elevated T1 signal of the RV free wall due to prolongation of the T1 relaxation time caused by myocardial edema (white arrows). Even greater prolongation is evident along the lateral pericardium (yellow arrows). e Increased T1 relaxation time along the mid and distal anterior LV wall (white arrows)

MyoMap software. Normal T1 and T1 values were obtained from series of normal patients to establish lab normal range (Normal T1 values were 910-930 msec and normal T2 values were 40-43 msec for our lab). More than 2 standard deviation (SD) above our normal range was considered elevated.

\section{Myocarditis versus acute coronary syndrome}

Due to concerning clinical presentation in certain patients, SARS-CoV-2 being a potential culprit for coronary plaque rupture and probability to cause myocardial ischemia with pleomorphic mechanisms, clinical judgment is a requisite in 
Fig. 4 Apical 2-chamber view in systole (image 2) and diastole (image 1) on TTE showing global LV dysfunction and apical 4- chamber view in systole (image 4) and diastole (image 3) showing the same. Coronary angiogram revealed patent epicardial vessels (image 5,6)
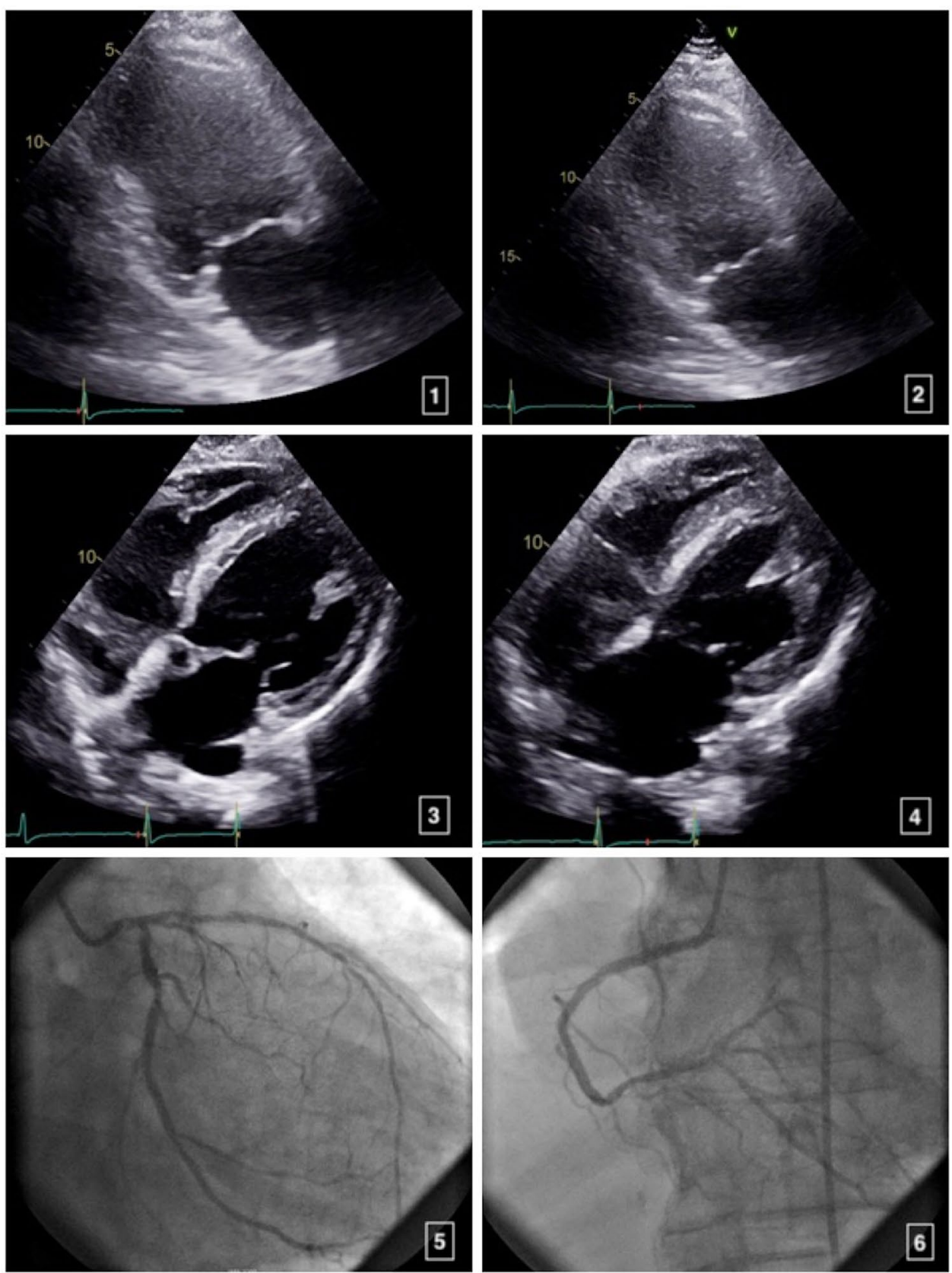

decision making as some patients may require invasive angiography to exclude acute coronary syndrome. In a New York City study of COVID-19 patients, 18 patients had ST-segment elevations on electrocardiogram, however, only 8 patients (44\%) had clinical myocardial infarction and only $6(33 \%)$ had evidence of obstructive coronary artery disease, whereas the remaining had non-coronary artery related myocardial injury; $13(72 \%)$ of these patients died during their hospitalization [48]. From our reviewed cases with COVID-19 myocarditis, 5 (42\%) had ST-segment elevation on initial electrocardiogram, of which 4 underwent coronary angiography and the diagnosis of acute coronary syndrome was excluded in all of them.
This has considerable ramifications as the discerning clinician must balance classic teachings with contemporary data both with far-reaching implications for balancing patient care with healthcare protections. In part, this was the impetus for our review and ultimate recommendations. 

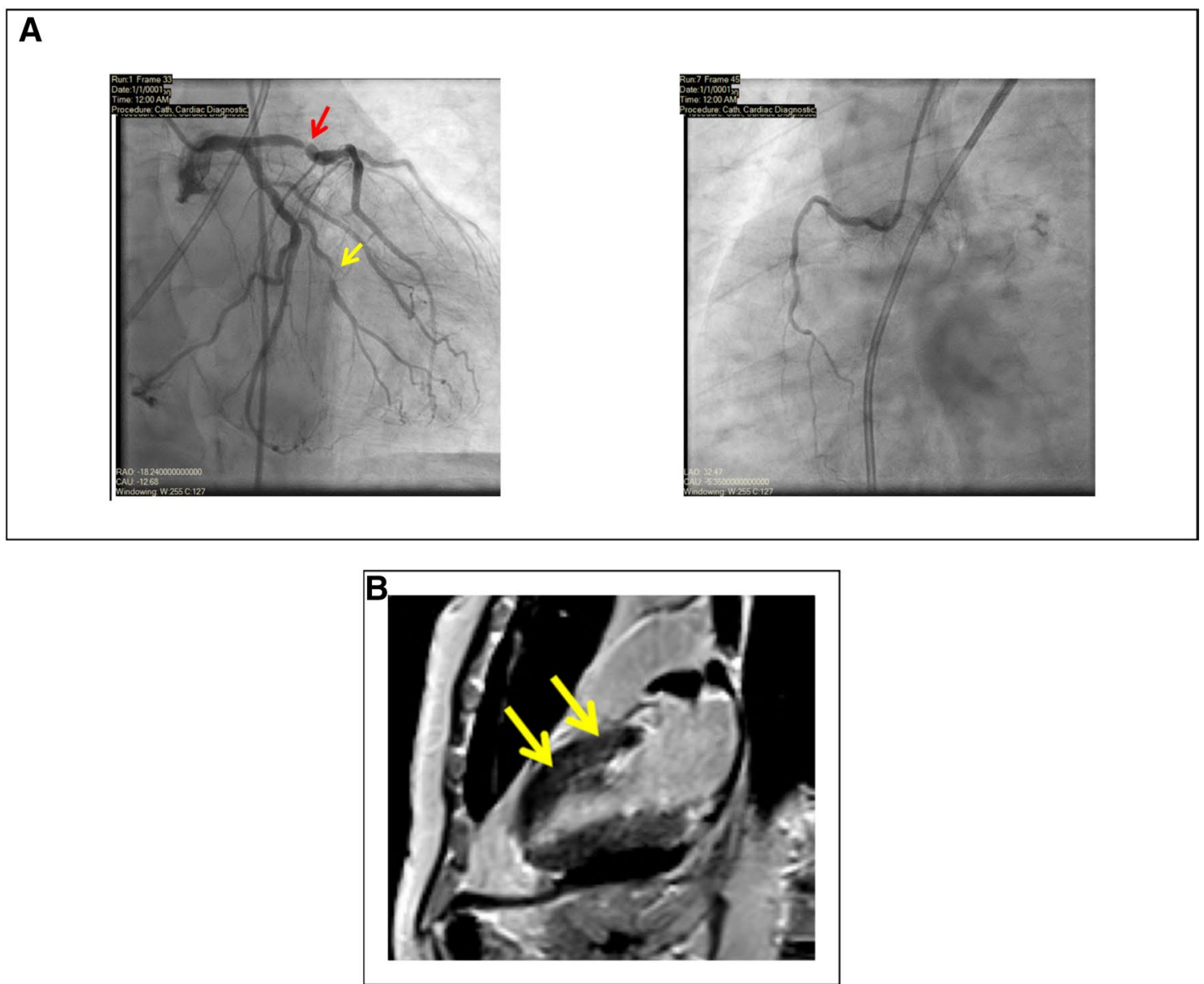

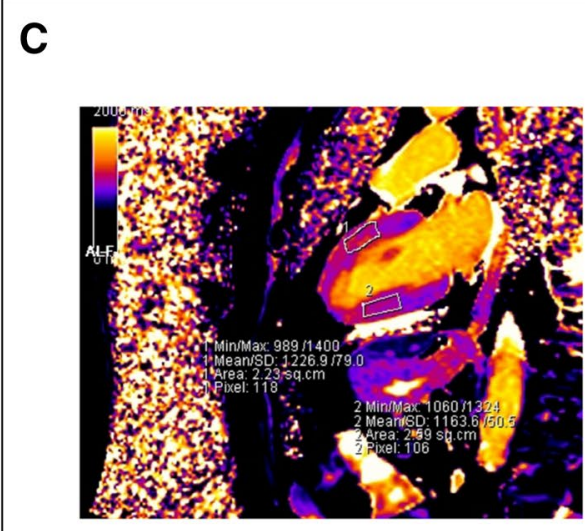

T1 Mapping

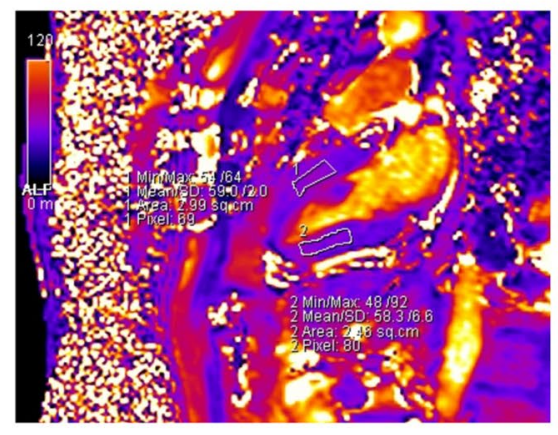

T2 Mapping
Fig. 5 a Coronary arteriography showing significant stenosis of the proximal LAD artery (red arrow) and 2nd obtuse marginal branch of circumflex artery (yellow arrow). b CMR with delayed gadolinium enhancement study showing viability of anterior LV wall (yellow arrow) but with a hint of more generalized myocardial edema. c T1 and T2 mapping technique revealing significant myocardial edema and prolonged $\mathrm{T} 1$ and $\mathrm{T} 2$ relaxation time 


\section{Outcomes of patients with COVID-19-related acute myocarditis}

It is consistently reported that patients with several cardiac comorbidities have much higher likelihood of having myocardial involvement. These patients are usually critically ill on presentation and suffer a high mortality rate [49]. A cohort study from Wuhan, China studying 416 hospitalized COVID-19 patients discovered that 82 patients (20\%) had acute myocardial injury and those patients had a very high mortality rate (42 patients, $51 \%$ ). When compared to patients without myocardial injury, patients with myocardial injury were significantly more likely to have cardiac comorbidities such as coronary artery disease $(29 \%$ vs. $6 \%)$ and heart failure (15\% vs. $2 \%)$ [50]. Another observational study of 187 COVID-19 hospitalized patients reported that $28 \%$ had evidence of myocardial injury. Overall mortality was $23 \%$ and was significantly higher among patients who had elevated troponin levels, especially in those with underlying cardiovascular comorbidities [49]. Additionally, a meta-analysis revealed that critically ill COVID-19 patients tend to have abnormal cardiac troponin levels and longitudinal monitoring of troponin levels might be able to predict those at risk for adverse clinical outcomes during the hospital admission [51]. Similarly, as described above, in one of our COVID19 patients with myocarditis we noticed a new, otherwise unexplained troponin elevation on routine daily blood work on day 10 of hospitalization. An echocardiogram on the same day revealed new LV wall motion abnormalities with reverse TakoTsubo pattern. This was followed by a rapid clinical deterioration and cardiac arrest within $24 \mathrm{~h}$.

It is also noteworthy that due to concerns for transmission of the virus to healthcare workers and other patients, the true incidence of acute imaging-confirmed myocarditis in COVID-19 patients is likely grossly underdiagnosed and underreported. However, based on ample evidence so far, myocarditis appears to be an adverse prognostic sign and is associated with rapid clinical deterioration in these patients. As more literature on COVID-19 myocarditis becomes available, we will be able to identify predictors of myocardial injury and hopefully, effective treatment options for these critically ill patients.

\section{Proposed CMR algorithm for COVID-19 patients}

Integrating the published and unpublished data along with our aggregate CMR experience [52], we propose the following algorithm to incorporate CMR in the suspected
COVID-19 patients with low to high pre-test probability myocarditis:

(1) If non-ST- elevation myocardial infarction (NSTEMI) via electrocardiogram and invasive /CT coronary angiogram is negative or mildly abnormal, in the setting of normal or abnormal TTE, consider CMR for myocarditis-edema imaging (See Fig. 6a).

(2) If ST-elevation myocardial infarction (STEMI) via electrocardiogram and invasive/CT coronary angiogram is negative or mildly abnormal, in the setting of normal or abnormal TTE, consider CMR for myocarditis-edema imaging (See Fig. 6b).

\section{Economic basis for upfront consideration for CMR}

The cost to the US healthcare system for inpatients with suspected or proven COVID-19 is fixed by DRG. However, for outpatients with suspected or proven COVID-19, the cost to the healthcare system of diagnostic imaging is a function of APC determined by Medicare. 2019 Medicare APC reimbursement amounts are provided in Table 1. Using the diagnostic flow chart suggested in Fig. 6, the total cost to the healthcare system to establish a diagnosis of myocarditis via the CMR strategy is $\$ 691.75$. Using the 2-D echo with stress diagnostic approach for an intermediate pre-test probability CAD patient, the cost to the healthcare system to establish a myocarditis diagnosis is $\$ 1686.73$. While cardiac MRI is capable of assessing structure, function, perfusion, inflammation and viability in one study, 2-D (with or without stress) echocardiographic-defined myocarditis is effectively a diagnosis of exclusion as it is not capable of assessing inflammation. Accordingly, the cardiac MRI imaging strategy additionally reduces cost to the healthcare system by $59 \%$ or $\$ 994.98$ per patient.

\section{Future directions}

To date, published trials have used the hard endpoints for morbidity and mortality. As intimated above, an imagingbased outcome might offer an improved understanding rooted on a pathophysiologic understanding of CMR-based edema imaging. Consequently, based on the known antiinflammatory effects of colchicine, a large (6000 patients) randomized, double-blind clinical trial in Montreal was initiated comparing colchicine vs. placebo in COVID 19 patients [53]. Extending this notion, based on the herein-described data and our experience, our CMR Research Group is initiating a randomized, double-blind two-arm clinical trial with planned enrollment of 400 COVID-19 patients within $72 \mathrm{~h}$ 
Fig. 6 a If a patient with suspected or proven COVID-19 presents with symptoms and signs of new right and/or left heart failure, assessment of cardiac biomarkers (troponin, pro-BNP, hs-CRP) helps to strategize diagnostic imaging. Cardiac MRI is not only capable of detecting myocardial ischemia and/or infarction, but also myocardial and pericardial inflammation. *excluding STEMI patients. b If coronary angiography shows no or only mild coronary atherosclerosis (CAD), cardiac MRI (CMR) can be pursued to assess possible myocarditis. If critical CAD is demonstrated, CMR prior to aortocoronary bypass would be appropriate to assess myocardial viability and possible myocarditis

\section{A \\ Role of Cardiac MRI in Evaluation of COVID-19 Patients}

\section{Clinical Evidence of Myocardial Dysfunction}

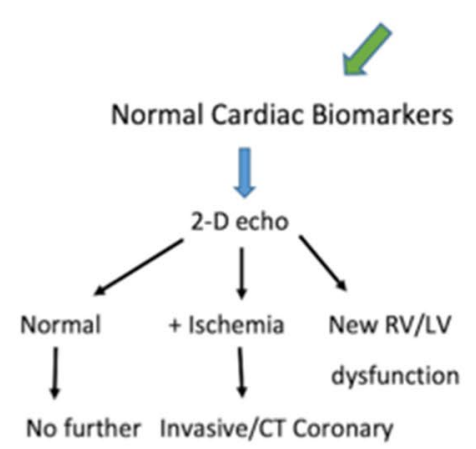

evaluation

angiogram

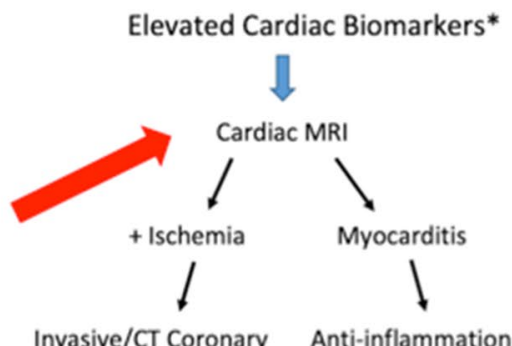

angiogram

therapy

\section{B Role of Cardiac MRI in STEMI Patients with Suspected or Proven COVID-19}

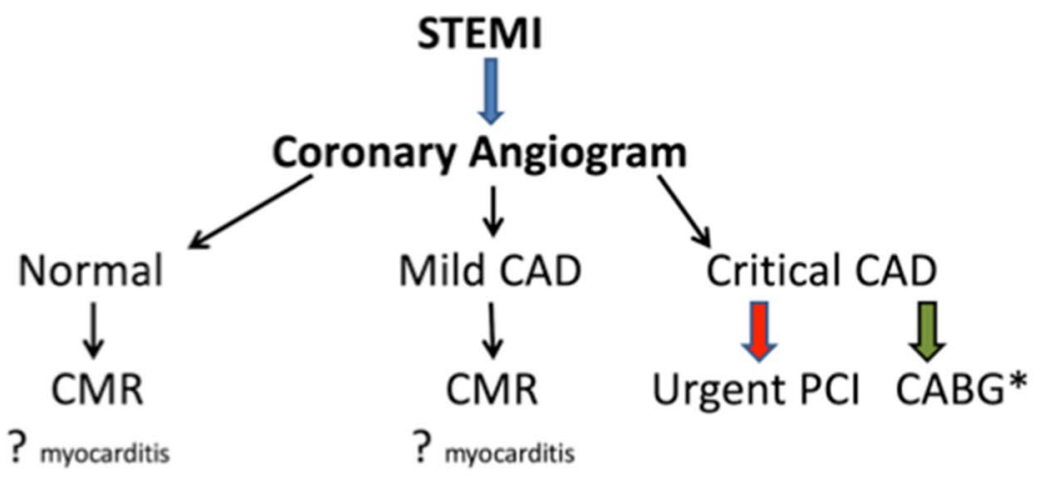

Table 1 Cost to the healthcare system per patient computed using 2019 Medicare Reimbursement values

\begin{tabular}{lll}
\hline Modality & APC & Reimbursement \\
\hline Nuclear stress/rest perfusion & 5593 & $\$ 1229.3$ \\
Cardiac MRI stress/rest perfusion & 5573 & $\$ 691.75$ \\
2-D echocardiogram with stress & 5524 & $\$ 994.98$ \\
Coronary CT angiography & 5571 & $\$ 201.74$ \\
\hline
\end{tabular}

of diagnosis, with trial arms of dexamethasone vs. colchicine (DEXCOL). This study will also assess cardiac biomarkers of troponin, pro-BNP and high sensitivity-CRP in all enrolled patients to assess the true incidence of myocarditis, confirmed with CMR, in COVID-19 positive patients. An interesting aspect of this trial is that it is designed to be a longitudinal study such that entry criteria is based on an outpatient diagnosis, not as traditionally tracked from an inpatient randomization schema. The basis for this nontraditional approach is that it provides the opportunity for a more fundamental understanding of COVID-19 over a longer period of time and an improved understanding for 
how morbidity and mortality may be affected by a focused attack on the inflammatory cascade with respect to several CMR-based assessments.

\section{Limitations}

Our review is limited by the evolving stage of this highly contagious virus, resulting in limited myocarditis data availability. Moreover, cases of COVID-19 myocarditis with available cardiac imaging are highly underreported, likely due to limited testing in order to limit the spread of the virus. Other limitations include the significant heterogeneity of patient selection, study designs and outcomes, which are at high risk to introduce bias. Due to the current pandemic, a significant amount of literature is being published in preprinted form without peer review, adding further limitation. Despite this, the inflammation cascade of COVID-19 triggered myocarditis is an important source of morbidity and mortality and is a source of major clinical and academic focus.

\section{Conclusions}

Acute myocarditis is relatively common in critically ill COVID-19 patients. The presentation of these patients is variable including LV systolic and diastolic dysfunction, arrhythmias and acute coronary syndrome. The majority of those cases can be sufficiently diagnosed with TTE, however CMR can be exceptionally helpful in many cases, especially equivocal cases where there is evidence of myocardial injury by cardiac biomarkers but inconclusive by TTE. CMR findings include myocardial edema seen in all patients with evidence of myocarditis, and notably, LGE is reported in the majority patients, consistent with myocardial fibrosis and suggests an independent mortality signal. Yet, we advise, these techniques should be performed with caution, according to the cardiovascular society guidelines, due to the risk of spreading the virus to healthcare workers and other patients as in all cardiac imaging procedures. Nonetheless, especially as the risk of myocarditis is higher in older patients and those with multiple cardiac comorbidities all associated with a very poor prognosis, we propose that advanced imaging, especially CMR can play a pivotal role in risk-prognostication for the COVID-19 patients.

Electronic supplementary material The online version of this article (https://doi.org/10.1007/s10554-020-02139-2) contains supplementary material, which is available to authorized users.

\section{Compliance with ethical standards}

Conflict of interest The authors declare that they have no conflict of interest.

\section{References}

1. Guo YR, Cao QD, Hong ZS, Tan YY, Chen SD, Jin HJ, Tan KS, Wang DY, Yan Y (2020) The origin, transmission and clinical therapies on coronavirus disease 2019 (COVID-19) outbreak-an update on the status. Mil Med Res 7(1):11. https://doi. org/10.1186/s40779-020-00240-0

2. CDC (2020) https://www.cdc.gov/coronavirus/2019-ncov/index .html Accessed 15 Dec 2020

3. Alhogbani T (2016) Acute myocarditis associated with novel Middle east respiratory syndrome coronavirus. Ann Saudi Med 36(1):78-80. https://doi.org/10.5144/0256-4947.2016.78

4. Huang C, Wang Y, Li X, Ren L, Zhao J, Hu Y, Zhang L, Fan G, Xu J, Gu X, Cheng Z, Yu T, Xia J, Wei Y, Wu W, Xie X, Yin W, Li H, Liu M, Xiao Y, Gao H, Guo L, Xie J, Wang G, Jiang R, Gao Z, Jin Q, Wang J, Cao B (2020) Clinical features of patients infected with 2019 novel coronavirus in Wuhan, China. Lancet 395(10223):497-506. https://doi.org/10.1016/S0140 $-6736(20) 30183-5$

5. Chen C, Zhou Y, Wang DW (2020) SARS-CoV-2: a potential novel etiology of fulminant myocarditis. Herz 45(3):230-232. https://doi.org/10.1007/s00059-020-04909-z

6. Inciardi RM, Lupi L, Zaccone G, Italia L, Raffo M, Tomasoni D, Cani DS, Cerini M, Farina D, Gavazzi E, Maroldi R, Adamo M, Ammirati E, Sinagra G, Lombardi CM, Metra M (2020) Cardiac involvement in a patient with coronavirus disease 2019 (COVID-19). JAMA Cardiol. https://doi.org/10.1001/jamacardio .2020 .1096

7. China. NHCotPsRo Chinese Clinical Guidance for COVID-19 Pneumonia Diagnosis and Treatment (7th edition). http://en.nhc. gov.cn/ Accessed 15 Dec 2020

8. Huang L, Zhao P, Tang D, Zhu T, Han R, Zhan C, Liu W, Zeng H, Tao Q, Xia L (2020) Cardiac involvement in recovered COVID-19 patients identified by magnetic resonance imaging. JACC Cardiovasc Imaging. https://doi.org/10.1016/j.jcmg.2020.05.004

9. Zheng YY, Ma YT, Zhang JY, Xie X (2020) COVID-19 and the cardiovascular system. Nat Rev Cardiol 17(5):259-260. https:// doi.org/10.1038/s41569-020-0360-5

10. Guzik TJ, Mohiddin SA, Dimarco A, Patel V, Savvatis K, MarelliBerg FM, Madhur MS, Tomaszewski M, Maffia P, D'Acquisto F, Nicklin SA, Marian AJ, Nosalski R, Murray EC, Guzik B, Berry C, Touyz RM, Kreutz R, Wang DW, Bhella D, Sagliocco O, Crea F, Thomson EC, McInnes IB (2020) COVID-19 and the cardiovascular system: implications for risk assessment, diagnosis, and treatment options. Cardiovasc Res. https://doi.org/10.1093/cvr/ cvaa106

11. Musher DM, Abers MS, Corrales-Medina VF (2019) Acute infection and myocardial infarction. N Engl J Med 380(2):171-176. https://doi.org/10.1056/NEJMra1808137

12. Chen L, Li X, Chen M, Feng Y, Xiong C (2020) The ACE2 expression in human heart indicates new potential mechanism of heart injury among patients infected with SARS-CoV-2. Cardiovasc Res 116(6):1097-1100. https://doi.org/10.1093/cvr/cvaa078

13. Caforio AL, Pankuweit S, Arbustini E, Basso C, Gimeno-Blanes J, Felix SB, Fu M, Helio T, Heymans S, Jahns R, Klingel K, Linhart A, Maisch B, McKenna W, Mogensen J, Pinto YM, Ristic A, Schultheiss HP, Seggewiss H, Tavazzi L, Thiene G, Yilmaz A, Charron P, Elliott PM, European Society of Cardiology Working 
Group on M, Pericardial D (2013) Current state of knowledge on aetiology, diagnosis, management, and therapy of myocarditis: a position statement of the European Society of Cardiology Working Group on Myocardial and Pericardial Diseases. Eur Heart J 34(33):2636-2648

14. Han Y, Chen T, Bryant J, Bucciarelli-Ducci C, Dyke C, Elliott MD, Ferrari VA, Friedrich MG, Lawton C, Manning WJ, Ordovas K, Plein S, Powell AJ, Raman SV, Carr J (2020) Society for Cardiovascular Magnetic Resonance (SCMR) guidance for the practice of cardiovascular magnetic resonance during the COVID19 pandemic. J Cardiovasc Magn Reson 22(1):26. https://doi. org/10.1186/s12968-020-00628-w

15. Skulstad H, Cosyns B, Popescu BA, Galderisi M, Salvo GD, Donal E, Petersen S, Gimelli A, Haugaa KH, Muraru D, Almeida AG, Schulz-Menger J, Dweck MR, Pontone G, Sade LE, Gerber B, Maurovich-Horvat P, Bharucha T, Cameli M, Magne J, Westwood M, Maurer G, Edvardsen T (2020) COVID-19 pandemic and cardiac imaging: EACVI recommendations on precautions, indications, prioritization, and protection for patients and healthcare personnel. Eur Heart J Cardiovasc Imaging. https://doi. org/10.1093/ehjci/jeaa072

16. Kirkpatrick JN, Mitchell C, Taub C, Kort S, Hung J, Swaminathan M (2020) ASE statement on protection of patients and echocardiography service providers during the 2019 novel coronavirus outbreak. J Am Coll Cardiol. https://doi.org/10.1016/j. jacc.2020.04.002

17. Mahmud E, Dauerman HL, Welt FG, Messenger JC, Rao SV, Grines C, Mattu A, Kirtane AJ, Jauhar R, Meraj P, Rokos IC, Rumsfeld JS, Henry TD (2020) Management of acute myocardial infarction during the COVID-19 pandemic. J Am Coll Cardiol. https://doi.org/10.1016/j.jacc.2020.04.039

18. Skali H, Murthy VL, Al-Mallah MH, Bateman TM, Beanlands R, Better N, Calnon DA, Dilsizian V, Gimelli A, Pagnanelli R, Polk DM, Soman P, Thompson RC, Einstein AJ, Dorbala S (2020) Guidance and best practices for nuclear cardiology laboratories during the coronavirus disease 2019 (COVID-19) pandemic: an Information Statement from ASNC and SNMMI. J Nucl Cardiol. https://doi.org/10.1007/s12350-020-02123-2

19. Biere L, Piriou N, Ernande L, Rouzet F, Lairez O (2019) Imaging of myocarditis and inflammatory cardiomyopathies. Arch Cardiovasc Dis 112(10):630-641. https://doi.org/10.1016/j. acvd.2019.05.007

20. Szekely Y, Lichter Y, Taieb P, Banai A, Hochstadt A, Merdler I, Gal Oz A, Rothschild E, Baruch G, Peri Y, Arbel Y, Topilsky Y (2020) The spectrum of cardiac manifestations in coronavirus disease 2019 (COVID-19) - a Systematic Echocardiographic Study. Circulation. https://doi.org/10.1161/CIRCULATIO NAHA. 120.047971

21. Cizgici AY, Zencirkiran Agus H, Yildiz M (2020) COVID-19 myopericarditis: It should be kept in mind in today's conditions. Am J Emerg Med. https://doi.org/10.1016/j.ajem.2020.04.080

22. Doyen D, Moceri P, Ducreux D, Dellamonica J (2020) Myocarditis in a patient with COVID-19: a cause of raised troponin and ECG changes. Lancet 395(10235):1516. https://doi.org/10.1016/ S0140-6736(20)30912-0

23. Hu H, Ma F, Wei X, Fang Y (2020) Coronavirus fulminant myocarditis saved with glucocorticoid and human immunoglobulin. Eur Heart J. https://doi.org/10.1093/eurheartj/ehaa190

24. Hua A, O'Gallagher K, Sado D, Byrne J (2020) Life-threatening cardiac tamponade complicating myo-pericarditis in COVID-19. Eur Heart J. https://doi.org/10.1093/eurheartj/ehaa253

25. Kim IC, Kim JY, Kim HA, Han S (2020) COVID-19-related myocarditis in a 21-year-old female patient. Eur Heart J. https://doi. org/10.1093/eurheartj/ehaa288

26. Kir D, Mohan C, Sancassani R (2020) HEART BRAKE-An unusual cardiac manifestation of Coronavirus disease 2019
(COVID-19). JACC Case Rep. https://doi.org/10.1016/j.jacca s.2020.04.026

27. Paul JF, Charles P, Richaud C, Caussin C, Diakov C (2020) Myocarditis revealing COVID-19 infection in a young patient. Eur Heart J Cardiovasc Imaging. https://doi.org/10.1093/ehjci/jeaa1 07

28. Sala S, Peretto G, Gramegna M, Palmisano A, Villatore A, Vignale D, De Cobelli F, Tresoldi M, Cappelletti AM, Basso C, Godino C, Esposito A (2020) Acute myocarditis presenting as a reverse Tako-Tsubo syndrome in a patient with SARS-CoV-2 respiratory infection. Eur Heart J. https://doi.org/10.1093/eurhe artj/ehaa286

29. Zeng JH, Liu YX, Yuan J, Wang FX, Wu WB, Li JX, Wang LF, Gao H, Wang Y, Dong CF, Li YJ, Xie XJ, Feng C, Liu L (2020) First case of COVID-19 complicated with fulminant myocarditis: a case report and insights. Infection. https://doi.org/10.1007/ s15010-020-01424-5

30. Coyle J, Igbinomwanhia E, Sanchez-Nadales A, Danciu S, Chu C, Shah N (2020) A recovered case of COVID-19 myocarditis and ARDS treated with corticosteroids, tocilizumab, and experimental AT-001. JACC Case Rep. https://doi.org/10.1016/j.jacca s.2020.04.025

31. Luetkens JA, Isaak A, Zimmer S, Nattermann J, Sprinkart AM, Boesecke C, Rieke GJ, Zachoval C, Heine A, Velten M, Duerr GD (2020) Diffuse myocardial inflammation in COVID-19 associated myocarditis detected by multiparametric cardiac magnetic resonance imaging. Circ Cardiovasc Imaging 13(5):e010897. https://doi.org/10.1161/CIRCIMAGING.120.010897

32. Beaudry JT, Dietrick B, Lammert DB, Constas A, McCaw J, Hammond J, Buendia M, Stein JE, Pekosz A, Schuette J, Mostafa HH, Hooper JE, Bernier M, Agwu A, Feldman LS (2020) Fatal SARS-CoV-2 inflammatory syndrome and myocarditis in an adolescent: a case report. Pediatr Infect Dis J. https://doi.org/10.1097/INF.0000000000002978

33. Caraffa R, Marcolongo R, Bottio T, Rizzo S, Bifulco O, Bagozzi L, D'Onofrio A, Caforio ALP, Jorgji V, Basso C, Gerosa G (2020) Recurrent autoimmune myocarditis in a young woman during the coronavirus disease 2019 pandemic. ESC Heart Fail. https://doi.org/10.1002/ehf2.13028

34. Othenin-Girard A, Regamey J, Lamoth F, Horisberger A, Glampedakis E, Epiney JB, Kuntzer T, de Leval L, Carballares M, Hurni CA, Rusca M, Pantet O, Di Bernardo S, Oddo M, Comte D, Piquilloud L (2020) Multisystem inflammatory syndrome with refractory cardiogenic shock due to acute myocarditis and mononeuritis multiplex after SARS-CoV-2 infection in an adult. Swiss Med Wkly 150:w20387. doi:https://doi.org/10.4414/ smw.2020.20387

35. Tiwary T, Baiswar S, Jinnur P (2020) A rare case of COVID19 myocarditis with cardiac tamponade in a young diabetic adult with renal failure. Cureus 12(11):e11632. https://doi. org/10.7759/cureus. 11632

36. Panchal A, Kyvernitakis A, Biederman R (2020) An interesting case of COVID-19 induced reversed takotsubo cardiomyopathy and insight on cardiac biomarkers. Cureus 12(11):e11296. https ://doi.org/10.7759/cureus.11296

37. Chen N, Zhou M, Dong X, Qu J, Gong F, Han Y, Qiu Y, Wang J, Liu Y, Wei Y, Xia J, Yu T, Zhang X, Zhang L (2020) Epidemiological and clinical characteristics of 99 cases of 2019 novel coronavirus pneumonia in Wuhan, China: a descriptive study. Lancet 395(10223):507-513. https://doi.org/10.1016/S0140 $-6736(20) 30211-7$

38. Lai CC, Shih TP, Ko WC, Tang HJ, Hsueh PR (2020) Severe acute respiratory syndrome coronavirus 2 (SARS-CoV-2) and coronavirus disease-2019 (COVID-19): The epidemic and the challenges. Int J Antimicrob Agents 55(3):105924. https://doi. org/10.1016/j.ijantimicag.2020.105924 
39. Saghazadeh A, Rezaei N (2020) Immune-epidemiological parameters of the novel coronavirus-a perspective. Expert Rev Clin Immunol. https://doi.org/10.1080/1744666X.2020.17509 54

40. Goitein O, Matetzky S, Beinart R, Di Segni E, Hod H, Bentancur A, Konen E (2009) Acute myocarditis: noninvasive evaluation with cardiac MRI and transthoracic echocardiography. AJR Am J Roentgenol 192(1):254-258. https://doi.org/10.2214/AJR.08.1281

41. Ferreira VM, Piechnik SK, Robson MD, Neubauer S, Karamitsos TD (2014) Myocardial tissue characterization by magnetic resonance imaging: novel applications of $\mathrm{T} 1$ and $\mathrm{T} 2$ mapping. J Thorac Imaging 29(3):147-154. doi:https://doi.org/10.1097/ RTI.0000000000000077

42. Friedrich MG (2008) Tissue characterization of acute myocardial infarction and myocarditis by cardiac magnetic resonance. JACC Cardiovasc Imaging 1(5):652-662. https://doi.org/10.1016/j. jcmg.2008.07.011

43. Wang Q, Fang P, He R, Li M, Yu H, Zhou L, Yu Y, Wang F, Rong Y, Zhang Y, Peng N, Lin Y, Lu M, Zhu Y, Peng G, Rao L, Liu S (2020) O-GlcNAc transferase promotes influenza A virus-induced cytokine storm by targeting interferon regulatory factor-5. Sci Adv 6(16):eaaz7086. https://doi.org/10.1126/sciadv.aaz7086

44. Deftereos S, Giannopoulos G, Papoutsidakis N, Panagopoulou V, Kossyvakis C, Raisakis K, Cleman MW, Stefanadis C (2013) Colchicine and the heart: pushing the envelope. J Am Coll Cardiol 62(20):1817-1825. https://doi.org/10.1016/j.jacc.2013.08.726

45. Robertson S, Martinez GJ, Payet CA, Barraclough JY, Celermajer DS, Bursill C, Patel S (2016) Colchicine therapy in acute coronary syndrome patients acts on caspase-1 to suppress NLRP3 inflammasome monocyte activation. Clin Sci (Lond) 130(14):12371246. https://doi.org/10.1042/CS20160090

46. Sabouraud A, Chappey O, Dupin T, Scherrmann JM (1994) Binding of colchicine and thiocolchicoside to human serum proteins and blood cells. Int J Clin Pharmacol Ther 32(8):429-432

47. Morgenstern D, Lisko J, Boniface NC, Mikolich BM, Mikolich JR (2016) Myocarditis and colchicine: a new perspective from cardiac MRI. J Cardiovasc Magn Reson 18(1):O100. https://doi. org/10.1186/1532-429X-18-S1-O100

48. Bangalore S, Sharma A, Slotwiner A, Yatskar L, Harari R, Shah B, Ibrahim H, Friedman GH, Thompson C, Alviar CL, Chadow HL, Fishman GI, Reynolds HR, Keller N, Hochman JS (2020) ST-segment elevation in patients with Covid-19-a case series. N Engl J Med. https://doi.org/10.1056/NEJMc2009020

49. Guo T, Fan Y, Chen M, Wu X, Zhang L, He T, Wang H, Wan J, Wang X, Lu Z (2020) Cardiovascular implications of fatal outcomes of patients with coronavirus disease 2019 (COVID-19). JAMA Cardiol. https://doi.org/10.1001/jamacardio.2020.1017

50. Shi S, Qin M, Shen B, Cai Y, Liu T, Yang F, Gong W, Liu X, Liang J, Zhao Q, Huang H, Yang B, Huang C (2020) Association of cardiac injury with mortality in hospitalized patients with COVID-19 in Wuhan, China. JAMA Cardiol. https://doi. org/10.1001/jamacardio.2020.0950

51. Lippi G, Lavie CJ, Sanchis-Gomar F (2020) Cardiac troponin I in patients with coronavirus disease 2019 (COVID-19): evidence from a meta-analysis. Prog Cardiovasc Dis. https://doi. org/10.1016/j.pcad.2020.03.001

52. Shah MB, Doyle M, Farah V, Biederman RWW (2018) Echocardiography and cardiovascular MRI entwined within the imaging domain; uniting the two. A compendium for the echocardiographer. Echocardiography 35(4):551-558. https://doi.org/10.1111/ echo. 13862

53. al. Te (2020) Colchicine coronavirus SARS-CoV2 Trial (COLCORONA) (COVID-19). Clinicaltrialsgov Identifier: NCT04322682

Publisher's Note Springer Nature remains neutral with regard to jurisdictional claims in published maps and institutional affiliations 